\title{
ARTICLES
}

\section{Effect of disorder on photonic band gaps}

\author{
M. M. Sigalas, C. M. Soukoulis, C. T. Chan,* R. Biswas, and K. M. Ho \\ Ames Laboratory, Department of Physics and Astronomy, and Microelectronics Research Center, \\ Iowa State University, Ames, Iowa 50011
}

(Received 24 August 1998; revised manuscript received 28 December 1998)

\begin{abstract}
We study the transmission of electromagnetic waves propagating in three-dimensional disordered photonic crystals that are periodic on the average with a diamond symmetry. The transmission has been calculated using the transfer matrix method. We study two different geometries for the scatterers: spheres and rods connecting nearest neighbors. We find that the gaps of the periodic structure survive to a higher amount of disorder in the rods' case than in the spheres' case. We argue that this is due to the connectivity of the rod structure that exists for any amount of disorder. [S0163-1829(99)04120-X]
\end{abstract}

\section{INTRODUCTION}

Photonic crystals are a class of artificially fabricated structures that have the ability to control and manipulate the propagation of electromagnetic (em) waves. Properly designed photonic crystals can prohibit the propagation of light, allow it only in certain frequency regions, or localize light in specified areas. They can be constructed in one, two, and three dimensions with either dielectric or/and metallic materials. ${ }^{1-3}$

Photonic band gaps can have a profound impact on many areas in pure and applied physics. Due to the absence of optical modes in the gap, spontaneous emission is suppressed for photons with frequencies in the forbidden region. It has been suggested that, by tuning the photonic band gap to overlap with the electronic band edge, electron-hole recombination can be controlled in a photonic band gap material, leading to enhanced efficiency and reduced noise in the operation of various optoelectronic devices. ${ }^{1-3}$ Besides technical applications in various areas, scientists are interested in the possibility of observing the localization of em waves by the introduction of defects and disorder in a photonic band gap material. ${ }^{4}$ This will be an ideal realization of the phenomenon of localization uncomplicated by many-body effects present in the case of electron localization.

In this paper we study the transmission properties of three dimensional photonic crystals that are periodic on the average. The photonic crystals have the diamond structure. The scatterers are either spheres or rods connecting nearest neighbors. In both cases there is a full band gap ${ }^{5}$ and this is the reason for studying diamond structure. Disorder has been introduced by disturbing the position of each lattice point. In addition, for the case of rods, we randomly break the connection of the dielectric network.

We use the transfer matrix method (TMM), introduced by Pendry and MacKinnon, ${ }^{6}$ to calculate the em transmission through a photonic crystal with defects. In the TMM the total volume of the photonic crystal is divided in small cells and the fields in each cell are coupled with those in the neighboring cells. Then the transfer matrix can be defined by relating the incident fields on one side of the photonic crystal to the outgoing fields on the other side. Using the TMM, the band structure of an infinite periodic system can be calculated, but the main advantage of this method is the calculation of transmission and reflection properties of em waves of various frequencies incident on a finite thickness slab of PBG material. In that case, the material is assumed to be periodic in the directions parallel to the interfaces. The TMM has previously been applied to defects in two dimensional photonic band gap (PBG) structures, ${ }^{7}$ photonic crystals with complex and frequency dependent dielectric constants, ${ }^{8}$ metallic PBG materials, ${ }^{9,10}$ and angular filters. ${ }^{11}$ In all these examples, the agreement between theoretical calculations and experimental measurements was very good.

\section{RESULTS AND DISCUSSION}

We study PBG materials with a diamond lattice symmetry. The $\mathbf{k}$ vector of the em waves is along the (001) direction and the thickness of the system along that direction is $L$ $\times a$ ( $a$ is the lattice constant of the diamond lattice, which is $1.27 \mathrm{~cm}$ in the following cases). In all the cases $L=20$. Along the (100) and (010) directions we use a supercell that contains $M$ conventional cubic cells; periodic boundary conditions are assumed along the edges of the supercell. The disorder $^{12}$ is introduced by moving the lattice points from their ideal positions in a diamond lattice, so the $x, y$, and $z$ components of the position of the $i$ th lattice point in the random system differ from those of the periodic case by $\gamma_{x} a$, $\gamma_{y} a$, and $\gamma_{z} a$, respectively; $\gamma_{x}, \gamma_{y}$, and $\gamma_{z}$ are random variables uniformly distributed over the interval $[-d, d]$. Due to computer time constraints, $\langle\ln (T)\rangle$ is calculated from an average over eight configurations. Each conventional unit cell is divided into $N \times N \times N$ points. The localization length is given by ${ }^{13,14}$ 


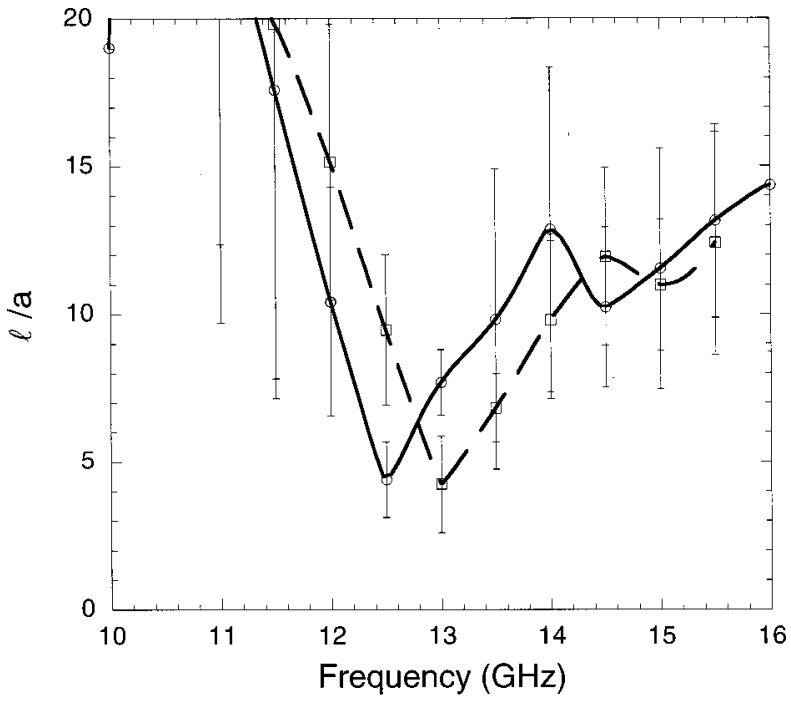

FIG. 1. Localization length $l$ vs frequency for a PBG with diamond lattice symmetry consisting of rods connecting nearest neighbors surrounded by air. The dielectric constant of the rods is 10 and their filling ratio is 0.25 . The lattice constant is $a=1.28 \mathrm{~cm}, M$ $=2, L=20$, and the strength of the disorder is $d=0.2$. Solid and dashed lines correspond to $N=7$ and 9 , respectively. (See the text for more details.)

$$
l=-\frac{2 L a}{\langle\ln (T)\rangle},
$$

where the $\langle\ln T\rangle$ is the logarithmic average of the transmission over eight different configurations.

We first study the convergence of our results relative to the number of subcells $N$. Figure 1 show the localization length for $N=7$ and 9 for rods connecting nearest neighbors in diamond lattice. In both cases the filing ratio of the dielectric rods is $f=0.25$, the dielectric constant $\epsilon=10, d / a$ $=0.2$, and $M=2$. The results for both $N=7$ and 9 are similar, except from a constant shift of $0.5 \mathrm{GHz}$. There is a minimum in both curves (Fig. 1) at 12.5 and $13 \mathrm{GHz}$ for $N=7$ and 9 , respectively. We can explain that difference by examining the corresponding periodic case (Fig. 2). The width of

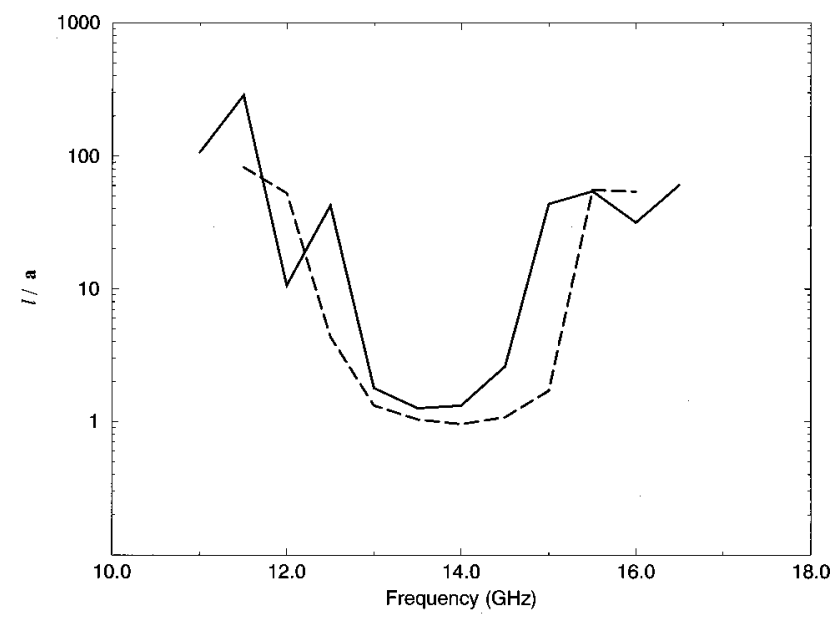

FIG. 2. Localization length $l$ vs frequency for a periodic photonic crystal similar to the one described in Fig. 1. Solid and dashed lines correspond to $N=7$ and 9 , respectively.

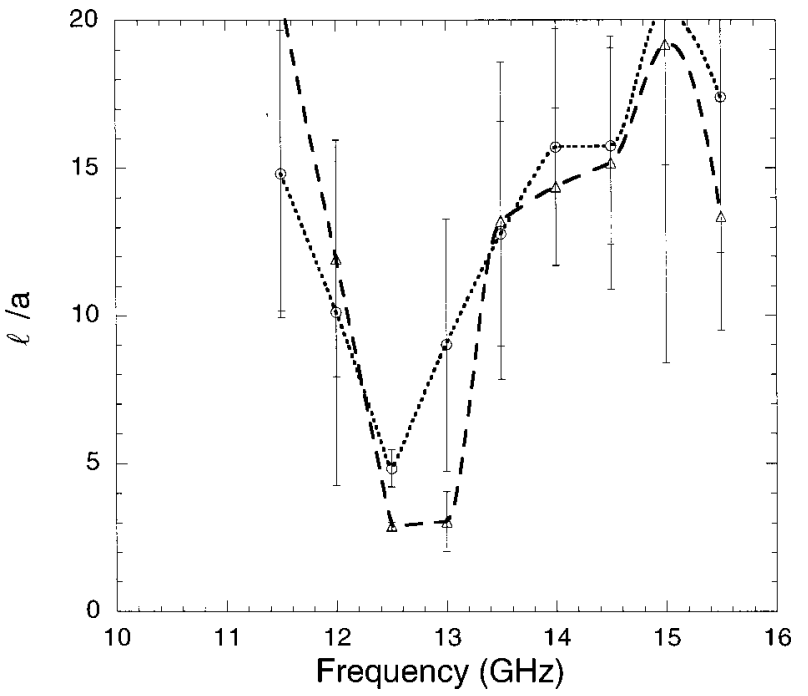

FIG. 3. Localization length $l$ vs frequency for a photonic crystal similar to the one described in Fig. 1. Dotted and dashed lines correspond to $d=0.2$ and 0.15 , respectively. In both cases, $M=3$ and $N=7$.

the gap is smaller for the $N=7$ case (compare the solid and dashed lines in Fig. 2). Also, the midgap frequency is at 13.5 and $14 \mathrm{GHz}$ for the $N=7$ and 9 cases, respectively. Apparently, the midgap frequency is smaller for the $N=7$ case and this is something that we observe even in the disorder case (compare the two lines in Fig. 1).

The error bars in the figures indicate the standard deviation of the localization length. The mean value of the localization length is in the center of the error bars. In the cases where part of the photonic band gap survives under disorder, the standard deviation of the localization length is small for frequencies inside the gap. In all the other cases, the standard deviation of the localization length is big, indicating big fluctuations of the transmission in different samples.

Since there are trivial differences between the $N=7$ and 9 cases that can be explained by comparing the corresponding periodic cases, we choose to work with the $N=7$ case. This will allow us to study even bigger supercells since the CPU time and the memory in the transfer matrix method depend on $(M \times N)^{6}$. By comparing the results for $M=2$ and 3 (solid line in Fig. 1 and dotted line in Fig. 3), we find that they are almost the same for frequencies around the minimum (at around $12.5 \mathrm{GHz}$ ). The localization length is longer for the $M=3$ case and for higher frequencies. However, the important point is the drop of the localization length at 12.5 $\mathrm{GHz}$, which is the reminiscent of the gap of the corresponding periodic case. This is consistent in both the $M=2$ and 3 cases. In fact, the localization length at the minimum is almost the same in both cases. It is really surprising that the gap survives to such a high amount of disorder. For a smaller amount of disorder $(d / a=0.15$; see the dashed line in Fig. 3 ), the drop of the localization length that is the remnant of the periodic gap is even more prominent. As we can see by comparing the solid and dotted lines in Fig. 3, the localization length at the minimum is smaller and the drop of the localization length is wider for the $d=0.15$ case than for the $d=0.2$ case. So, as we expect, the distortion of the photonic band gap due to the disorder decreases as the strength of the disorder decreases. 


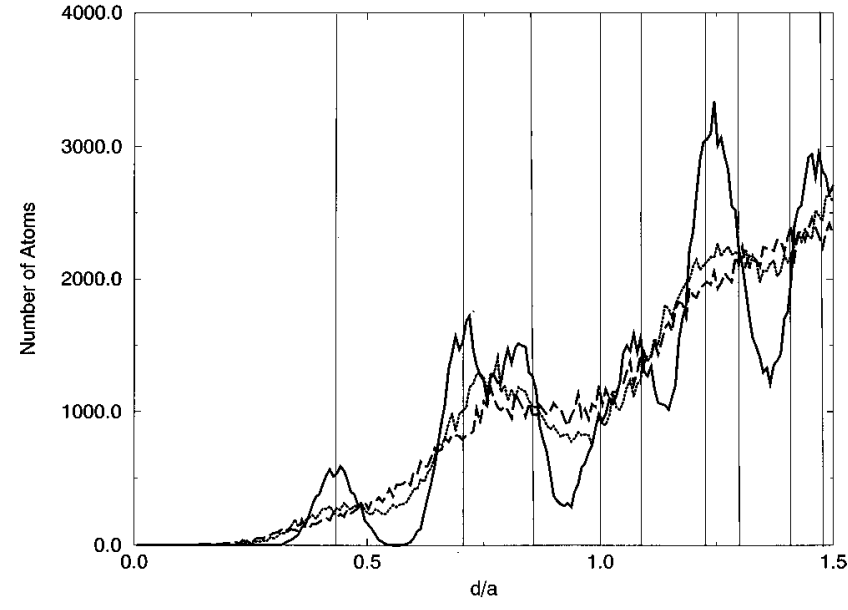

FIG. 4. Average number of neighboring atoms vs the separation distant $D$ normalized to the lattice constant $a$. The structure is diamond with $d=0.05,0.1$, and 0.15 (solid, dotted, and dashed lines, respectively). The vertical lines indicate the locations of the neighbors in a periodic diamond lattice.

In order to show how unexpected the survival of the gap to such a high amount of disorder was, we calculate the average number of neighbors in a distorted diamond lattice as a function of the distance $D$ from a lattice point. For $d$ $=0.05$ (solid line in Fig. 4), the first three peaks correspond to the locations of the first three nearest neighbors in the corresponding periodic case. For $d=0.1$ (dotted line in Fig. 4), the first peak at $D / a=0.433$ has almost disappeared and only a small peak appears between the second and third nearest neighbors $(D / a=0.8)$. For $d=0.15$ (dashed line in Fig. 4), all the peaks have disappeared. That means that for $d$ $>0.1$, any notion of nearest neighbors has been eliminated. So the existence of the gap for such high amounts of disorder $(d=0.2)$ is not related to the nearest neighbor interaction effects as in the case of electrons in disordered semiconductors, such as $\mathrm{Si}$. In that case it has been suggested that although the atoms in the semiconductor have random positions, the tetrahedral symmetry is still present. This is due to the interaction of the electrons in one atom with the electrons in the first neighboring atoms and this is the reason for the existence of the gap in semiconductors even in their disordered phases. Here, in the electromagnetic case, the existence of the gap is related to the connectivity of the high dielectric material. Note that the connectivity of the structure exists in any amount of disorder. Earlier studies suggested that periodic structures with network topology (connected structures) give rise to wider gaps for electromagnetic waves. ${ }^{15,16}$ For scalar and elastic waves the cermet topology (isolated scatterers) favors the formation of band gaps. ${ }^{15,16}$

We have also studied photonic crystals with diamond symmetry consisting of air spheres in a material with dielectric constant 10. The filling ratio of the air spheres is 0.77 , $N=7$, and $L=20$. Figure 5 shows the localization length for $d=0.05$. There is good convergence between the results for $M=2$ and 3 (compare the solid and dotted lines in Fig. 5). There is a minimum of $l$ at around $14 \mathrm{GHz}$, which is the remnant of the gap of the corresponding periodic case. However, by further increasing the disorder the gap disappears. Figure 6 shows the localization length $l$ as a function of the

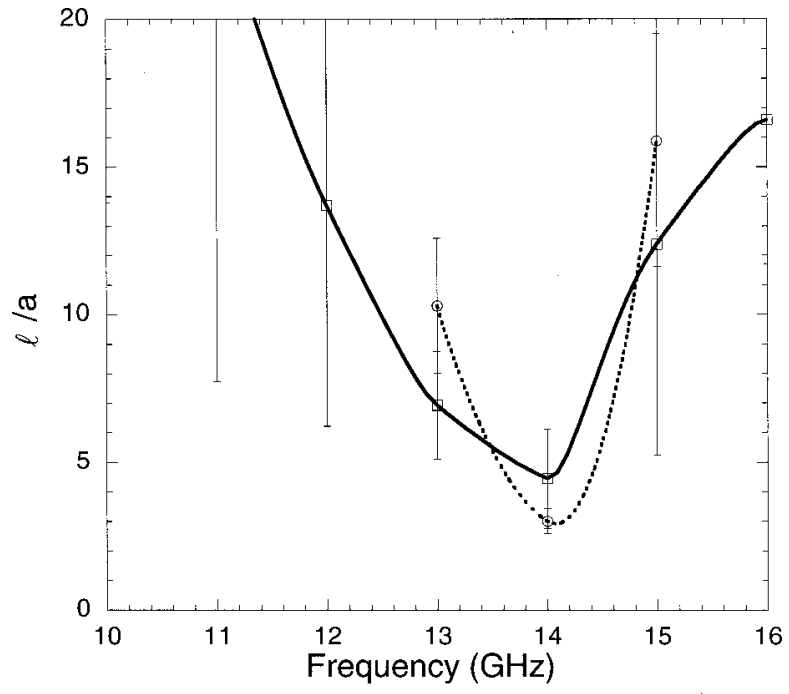

FIG. 5. Localization length vs frequency for a photonic crystal with diamond lattice symmetry consisting of air spheres with filling ratio $f=0.77$ in a material with dielectric constant 10 . The lattice constant is $a=1.28 \mathrm{~cm}$ and the strength of the disorder is $d$ $=0.05$. $M=2$ and 3 for solid and dotted lines, respectively.

frequency for two different widths $M=2$ and 3 ; in both cases $d=0.1$. Although the results are not very convergent relative to the width $M$ (except for the frequency $12 \mathrm{GHz}$ ), it is clearly seen that the gap, which appears between 12.8 and 14.2 GHz in the periodic case, has almost disappeared. In its place a region of localized states has appeared with a localization length of around $12 a$. Also note that the the standard deviation of the localization length is high for almost all the frequencies. This is one more indication that states exist in the region where the photonic band gap of the periodic case used to be. The reason for the disappearance of the gap to such a small amount of disorder relative to the previous connected structures is related to the lack of connectivity of the the high dielectric material. By disturbing the positions of the lattice points, the air spheres start to overlap (in that case the shape of air inclusions is no longer spherical). That creates some voids in the structure and eventually the dielectric

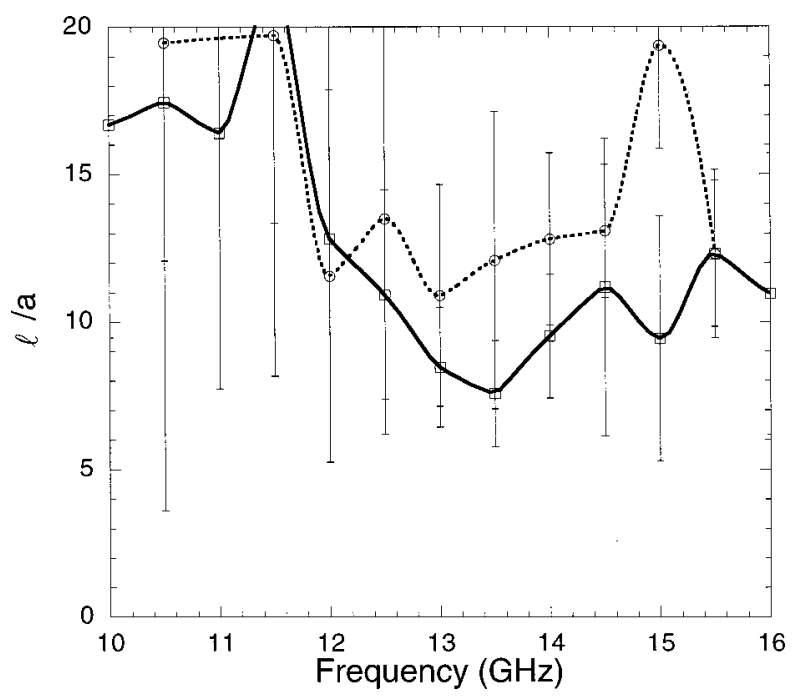

FIG. 6. Same as in Fig. 5 for $d=0.1$. 


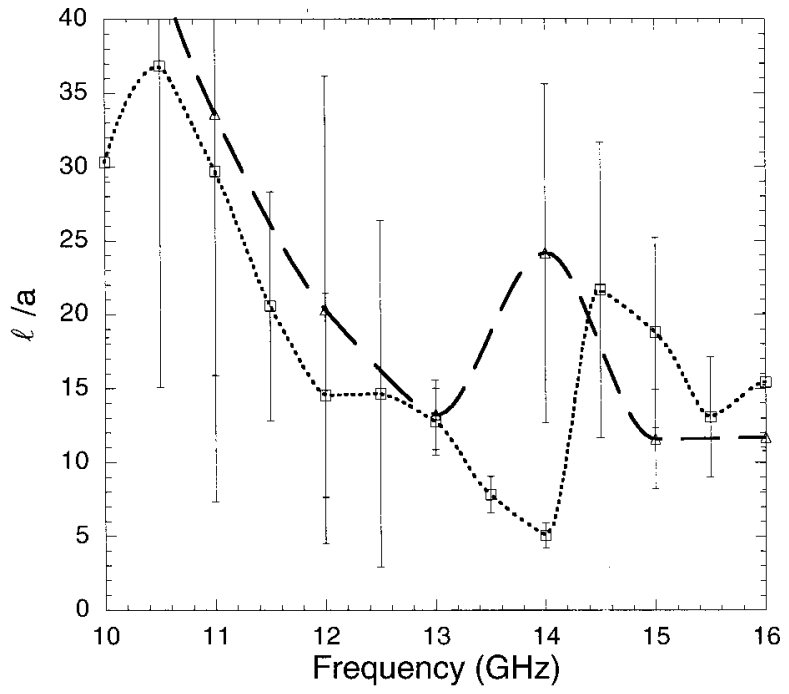

FIG. 7. Localization length vs frequency for a photonic crystal with diamond lattice symmetry consisting of rods connecting nearest neighbors surrounded by air. The dielectric constant of the rods is 10 and their filling ratio is 0.25 . The lattice constant is $a$ $=1.28 \mathrm{~cm}, M=2, L=20$, and $N=7$. The bonds are broken in $30 \%$ and $50 \%$ of the lattice points (dotted and dashed lines, respectively).

network breaks. We also studied isolated dielectric spheres with filling ratio 0.34 embedded in air. In the periodic case, there is a full band gap. ${ }^{5}$ However, similarly to the air spheres' case, the gap disappears for $d$ around 0.1 .

Finally, returning to the rods' case, we study the behavior of the localization length when the connectivity of the structure is broken. The lattice points remain in their periodic locations, but now we remove the dielectric material that is inside a sphere of radius $0.18 a$. The center of this sphere coincides with some lattice points that are chosen randomly. Again, the localization length is calculated by finding the logarithmic average of the transmission over eight configurations. By randomly breaking the network at 30\% of the lattice points (dotted line in Fig. 7), a dip in the localization length appears between 12.5 and $14.5 \mathrm{GHz}$, which is the reminiscent of the gap of the periodic case. However, by randomly breaking the dielectric network at $50 \%$ of the lattice points (dashed line in Fig. 7), the dip disappears. This is one more indication of the importance of the connectivity of the dielectric material in the existence of the gap to a high amount of disorder.

\section{CONCLUSION}

We studied the transmission of electromagnetic waves propagating into disordered photonic crystals that are periodic on the average. The periodic crystal has diamond symmetry. Two different geometries were studied: spheres and dielectric rods connecting nearest neighbors. Disorder was introduced by randomly displacing the lattice points from their periodic positions. We found that the gaps of the periodic case survive for a high amount of disorder in the geometry of rods. For spheres, the gaps disappear at almost half the amount of disorder than for the rods' case. We attribute this to the connectivity of the dielectric material in the rods' geometry that exists for any amount of disorder.

\section{ACKNOWLEDGMENTS}

This work was made possible in part by the Scalable Computing Laboratory, which is funded by Iowa State University and Ames Laboratory. Ames Laboratory is operated by the U.S. Department of Energy by Iowa State University under Contract No. W-7405-Eng-82.
*Permanent address: Department of Physics, Hong Kong University of Science and Technology, Hong Kong, China.

${ }^{1}$ See J. Opt. Soc. Am. B 10 (2), (1993), special issue on the development and applications of materials exhibiting photonic band gaps, edited by C. M. Bowden, J. P. Dowling, and H. O. Everitt; see also Photonic Band Gaps and Localization, Proceedings of the NATO ARW, edited by C. M. Soukoulis (Plenum, New York, 1993); for a more recent review see the articles in Photonic Band Gap Materials, edited by C. M. Soukoulis (Kluwer, Dordrecht, 1996).

${ }^{2}$ J. D. Joannopoulos, R. D. Meade, and J. N. Winn, Photonic Crystals (Princeton University Press, Princeton, 1995).

${ }^{3}$ E. Yablonovitch, Phys. Rev. Lett. 58, 2059 (1987).

${ }^{4}$ S. John, Phys. Rev. Lett. 58, 2486 (1987); S. John, Comments Condens. Matter Phys. 14, 193 (1988).

${ }^{5}$ K. M. Ho, C. T. Chan, and C. M. Soukoulis, Phys. Rev. Lett. 65 , 3152 (1990); C. T. Chan, K. M. Ho, and C. M. Soukoulis, Europhys. Lett. 16, 563 (1991).

${ }^{6}$ J. B. Pendry and A. MacKinnon, Phys. Rev. Lett. 69, 2772 (1992); J. B. Pendry, J. Mod. Opt. 41, 209 (1994).
${ }^{7}$ M. M. Sigalas, C. M. Soukoulis, E. N. Economou, C. T. Chan, and K. M. Ho, Phys. Rev. B 48, 14121 (1993).

${ }^{8}$ M. M. Sigalas, C. M. Soukoulis, C. T. Chan, and K. M. Ho, Phys. Rev. B 49, 11080 (1994).

${ }^{9}$ M. M. Sigalas, C. T. Chan, K. M. Ho, and C. M. Soukoulis, Phys. Rev. B 52, 11744 (1995).

${ }^{10}$ J. S. McCalmont, M. M. Sigalas, G. Tuttle, K. M. Ho, and C. M. Soukoulis, Appl. Phys. Lett. 68, 2759 (1996).

${ }^{11}$ M. M. Sigalas, J. S. McCalmont, K. M. Ho, and G. Tuttle, Appl. Phys. Lett. 68, 3525 (1996).

${ }^{12}$ M. M. Sigalas, C. M. Soukoulis, C.-T. Chan, and D. Turner, Phys. Rev. B 53, 8340 (1996).

${ }^{13}$ C. M. Soukoulis, I. Webman, G. S. Grest, and E. N. Economou, Phys. Rev. B 26, 1838 (1982).

${ }^{14}$ A. McKinnon and B. Kramer, Z. Phys. B 53, 1 (1983).

${ }^{15}$ E. N. Economou and M. M. Sigalas, Phys. Rev. B 48, 13434 (1993).

${ }^{16}$ M. M. Sigalas, E. N. Economou, and M. Kafesaki, Phys. Rev. B 50, 3393 (1994). 